\title{
Asymptotics for the Probability of Connectedness and the Distribution of Number of Components
}

\author{
Jason P. Bell \\ Department of Mathematics \\ University of California, San Diego \\ La Jolla, CA 92903-0112, USA \\ (email: jbell@math.ucsd.edu) \\ Edward A. Bender \\ Department of Mathematics \\ University of California, San Diego \\ La Jolla, CA 92903-0112, USA \\ (email: ebender@ucsd.edu) \\ Peter J. Cameron \\ School of Mathematical Sciences \\ Queen Mary and Westfield College \\ Mile End Road \\ London E1 4NS, England \\ (email: p.j.cameron@qmw.ac.uk) \\ L. Bruce Richmond \\ Department of Combinatorics and Optimization \\ University of Waterloo \\ Waterloo, Ontario N2L 3G1, Canada \\ (email: lbrichmond@math.uwaterloo.ca)
}

Submitted: January 21, 2000

Accepted: May 30, 2000

\begin{abstract}
Let $\rho_{n}$ be the fraction of structures of "size" $n$ which are "connected"; e.g., (a) the fraction of labeled or unlabeled $n$-vertex graphs having one component, (b) the fraction of partitions of $n$ or of an $n$-set having a single part or block, or (c) the fraction of $n$-vertex forests that contain only one tree. Various authors have considered $\lim \rho_{n}$, provided it exists. It is convenient to distinguish three cases depending on the nature of the power series for the structures: purely formal, convergent on the circle of convergence, and other. We determine all possible values for the pair $\left(\lim \inf \rho_{n}, \lim \sup \rho_{n}\right)$ in these cases. Only in the convergent case can one have $0<\lim \rho_{n}<1$. We study the existence of $\lim \rho_{n}$ in this case.
\end{abstract}

AMS-MOS Subject Classification (1990): 05A16; Secondary: 05C30, 05C40 


\section{Introduction}

Throughout, $A_{n}$ will denote the number of structures of size $n, C_{n}$ will denote the number that are connected, and $\rho_{n}=C_{n} / A_{n}$ whenever $A_{n} \neq 0$. We consider two situations: either the objects are labeled and the exponential generating functions are related by

$$
A(x)=\exp (C(x))
$$

or the objects are unlabeled and the ordinary generating functions are related by

$$
A(x)=\exp \left(\sum_{k \geq 1} C\left(x^{k}\right) / k\right) .
$$

Perhaps the most interesting omissions are

- objects with "noncrossing" parts, which lead to functional equations as in Beissinger [2] and Flajolet and Noy [12], and

- multiplicative objects, which lead to Dirichlet series.

We are interested in the three asymptotic probabilities

$$
\rho_{\text {inf }}=\liminf \rho_{n}, \quad \rho_{\text {sup }}=\limsup \rho_{n}, \quad \text { and } \quad \rho=\lim \rho_{n}
$$

where the limits are taken through those $n$ for which $A_{n} \neq 0$ and $\rho$ is defined only when that limit exists.

When $C(x)$ is a polynomial, we immediately have $\rho=0$.

Therefore we assume that $C(x)$ is not a polynomial.

Information on possible values for $\rho_{\text {inf }}$ and $\rho_{\text {sup }}$ are given in Theorem 1.

Various authors have obtained results about when $\rho$ exists. See the papers by Compton [10], Knopfmacher and Knopfmacher [18] and Bender, Cameron, Odlyzko and Richmond [5]. Related to this is the question of whether first-order limit laws exist [6-8,21].

If $\rho$ exists, one may ask about various limiting probability distributions. Perhaps the three most interesting questions are as follows.

- What, if any, is the limiting behavior of probability distribution of the number of components when objects of size $n$ are selected uniformly at random? More on this shortly.

- What, if any, is the limiting behavior of the joint distribution of objects of various sizes? We do not discuss this. See Arratia, Barbour, and Tavaré [1] for some results.

- What, if any, is the limiting behavior of probability distribution of the size of the largest component when objects of size $n$ are selected uniformly at random? We do not discuss this. See Gourdon [14] for some results.

The limiting distribution of the number of components, when it exists, has four common behaviors. 
Let $0 \leq R \leq \infty$ be the radius of convergence of $C(x)$. If $R=0$, the mass of the distribution is concentrated at 1 because $C_{n} / A_{n} \rightarrow 1$ by Theorem 1 . If $C(R)$ diverges, the distribution is often normal. Arguments for normality usually rely on analytic properties of the generating function as in Flajolet and Soria [13]. When there is a logarithmic singularity on the circle of convergence, Hwang [17] obtained refinements which are similar to what happens when $C(R)$ converges: The labeled case leads to a shifted Poisson and unlabeled case is more complicated. Compton [10] obtained some results in these cases, and we present additional ones in Theorem 2. In contrast to the analytic approaches for normality, our method relies on direct estimations of sums.

We thank A. Meir for helpful comments and references.

\section{Results and Discussion}

It is useful to consider cases depending on $R$ and the convergence of $C(R)$. With $\mathrm{L}$ for labeled and $\mathrm{U}$ for unlabeled:

$$
\begin{array}{lll}
\text { L0 or U0 } & \text { means } & R=0, \\
\text { LD or UD } & \text { means } & R>0 \text { and } C(R) \text { diverges, } \\
\text { LC or UC } & \text { means } & R>0 \text { and } C(R) \text { converges. }
\end{array}
$$

For case LD we may have $R=\infty$. Since $C_{n}$ counts objects, it is an integer and so, in the unlabeled case, $R \leq 1$ and $C(1)$ diverges. From (3) and the fact that $C_{n}$ is an integer, we have $R<1$ for case UC.

Theorem 1. The following three cases completely describe all possibilities for the pair $\rho_{\text {inf }}, \rho_{\text {sup }}$, subject to the obvious constraint that $0 \leq \rho_{\text {inf }} \leq \rho_{\text {sup }} \leq 1$.

(a) For LO and UO, $\rho_{\mathrm{sup}}=1$ and all values are possible for $\rho_{\mathrm{inf}}$.

(b) For $L D$ and $U D, \rho_{\mathrm{inf}}=0$ and all values are possible for $\rho_{\mathrm{sup}}$.

(c) For $L C$ and $U C$, all values are possible for the pair $\left(\rho_{\mathrm{inf}}, \rho_{\mathrm{sup}}\right)$ except $(0,0)$ and $(1,1)$.

These results still hold if we also require that $C_{n} \neq 0$ for all sufficiently large $n$.

"All values are possible" means that for each possible $R$ in each of the six cases and for any possible value, there exist nonnegative integers $C_{n}$ so that the value occurs. We immmediately have the following corollaries.

Corollary 1.1. Only $\left(\rho_{\text {inf }}, \rho_{\text {sup }}\right)=(0,1)$ can occur in all six cases.

Corollary 1.2. If $\rho$ exists, then

(a) for LO and $U 0 \rho=1$,

(b) for $L D$ and $U D \rho=0$,

(c) for $L C$ and $U C$ any value in the interval $(0,1)$ is possible.

For any power series $F(x)$, let $f_{n}=\left[x^{n}\right] F(x)$. (Thus $c_{n}=C_{n} / n$ ! in the labeled case and $c_{n}=C_{n}$ in the unlabeled case.) Let $c_{n}^{(k)}=\left[x^{n}\right] C(x)^{k}$. There is a close relation between the existence of $\rho$ and the statement $\lim _{n \rightarrow \infty} c_{n}^{(2)} / c_{n}=2 C(R)$ : 
- Suppose $R=0$. We know by Theorem 1 (a) that $\rho=1$ if it exists. Wright $[25,26]$ proved that $\rho=1$ if and only if $c_{n}^{(2)} / c_{n} \rightarrow 0$. Since $R=0, C(R)=0$.

- When $C(R)$ diverges, the conditions are not equivalent. Cameron [9] proved that $c_{n}^{(2)} / c_{n} \rightarrow \infty$ implies $\rho=0$, but the converse is false. To see this for LD (UD is similar), let $C_{n}=n ! 2^{n}$ if $n$ is a perfect square and $C_{n}=1$ otherwise. If $p$ is a prime congruent to 3 modulo 4 and $n=p^{2}$, then $n$ is not the sum of two nonzero squares and so at most one of $c_{k}$ and $c_{n-k}$ exceeds 1 . Thus

$$
c_{n}^{(2)} \leq n+2 \sum_{k^{2}<n} 2^{k^{2}} \leq n+2 n 2^{(p-1)^{2}}=o\left(c_{n}\right) .
$$

If $\sigma_{k}(n)$ is the number of ways to write $n$ as a sum of $k$ nonzero squares, then $k ! a_{n} \geq$ $c_{n}^{(k)} \geq \sigma_{k}(n) c_{n}$. Since $\liminf \inf _{n \rightarrow \infty} \sigma_{k}(n)=\infty$ for sufficiently large $k$, we have $\rho=0$.

- In cases LC and UC, the next theorem proves the equivalence of the conditions under the additional assumption that $\lim c_{n-1} / c_{n}$ exists.

Theorem 2. Suppose that $R>0$ and $C(R)$ converges. Let $A(x, y)$ enumerate structures by size and number of components. Thus

$$
A(x, y)= \begin{cases}\exp (y C(x)) & \text { for labeled structures } \\ \exp \left(\sum_{k \geq 1} y^{k} C\left(x^{k}\right) / k\right) & \text { for unlabeled structures. }\end{cases}
$$

Suppose that

(a) $c_{n}>0$ for all sufficiently large $n$ and

(b) $\lim c_{n-1} / c_{n}$ exists (it will be $R$ ).

Then the following statements are equivalent:

(c) $\sum c_{k} c_{n-k} \sim 2 C(R) c_{n}$.

(d) If $\omega=\omega(n) \rightarrow \infty$, then $\sum_{k=\omega}^{n-\omega} c_{k} c_{n-k}=o\left(c_{n}\right)$.

(e) $\rho$ exists. (By Theorem 1, we then have $\rho>0$.)

(f) If the number of components in a random structure is $X_{n}$, then for each fixed $d>0$

$$
\operatorname{Pr}\left(X_{n}=d\right) \sim \frac{\left[y^{d-1}\right] A(R, y)}{A(R, 1)}
$$

and

$$
\mathrm{E}\left(X_{n}\right) \sim 1+\left.\frac{\partial A(R, y)}{A(r, 1) \partial y}\right|_{y=1}
$$

In particular, with $d=1$ in (4), we have $\rho=1 / A(R, 1)=1 / A(R)$.

It may be possible to improve the theorem. In particular, we make the following conjectures.

Conjecture 1. Theorem 2(b) can be replaced by

(b') $\lim a_{n-1} / a_{n}$ exists (it will be $R$ ).

Conjecture 2. The existence of $\rho$ implies (b). 
Some comments on the conditions in the theorem are in order:

- The equivalence of (c) and (e) in the labeled case was given by Embrechts [11] in a more general context involving probability measures. (A subexponential measure satisfying (b) and (c) is said to belong to $S D(R)$.)

- In the labeled case, (f) asserts that $X_{n}-1$ is asymptotically Poisson with $\lambda=C(R)$.

- Conditions (a) and (b) are not sufficient to deduce the existence of $\rho$. To see this, define

$$
c_{n}=\left[2^{n} / n^{d(n)}\right] \quad \text { where } \quad d(n)=2+9 \min _{k}\left|n-2^{k}\right| / n .
$$

Then $c_{n-1} / c_{n} \sim 1 / 2$ and $c_{n}=O\left(2^{n} / n^{2}\right)$. Hence $R=1 / 2$ and $C(R)$ converges. Let $m=2^{k}$ and note that $c_{m} \sim 2^{m} / m^{2}, c_{2 m} \sim 2^{2 m} /(2 m)^{2}$, and $c_{3 m} \sim 2^{3 m} /(3 m)^{5}$. Hence $c_{3 m}=o\left(c_{m} c_{2 m}\right)$ in violation of $(\mathrm{d})$. Rudin [23, p.990] constructs a log-convex counterexample.

- When $\lim c_{n-1} / c_{n}$ exists, perturbing the $c_{n}$ 's by $o\left(c_{n}\right)$ does not affect the existence of $\rho$. To see this, note that the perturbations do not affect the validity of (b) or (d) in the theorem.

Knopfmacher and Knopfmacher's [18] abstract prime number theorem for additive semigroups follows from case UC of Theorem 2 since (a), (b), and (d) are easily verified.

Compton [10] dealt with LC and UC in his Theorems 10 and 11, respectively. He allowed either (a) $c_{n-1} / c_{n} \rightarrow R$ or (b) $a_{n-1} / a_{n} \rightarrow R$. Our theorem is stronger than Compton's (a) and would be stronger than his (b) if Conjecture 1 is true. To see that our theorem is stronger than Compton's (a), first note that our theorem applies to

$$
a_{n}=\left[\frac{x(n)}{R^{n} n^{\ln n}}\right] \text { where } x(n)=1 \text { for } \mathrm{UC} \text { and } x(n)=n ! \text { for LC, }
$$

but his does not. Second, note that our (c) and hence his Theorems 10 and 11 follow from the last sentence in his Lemma 9 by setting $\alpha(x)=\beta(x)=\delta(x)=C(x)$. Although some of Compton's results are weaker than ours, they may be easier to apply since verifying (c) or (d) in our theorem may be difficult. In this connection, it should be noted that Lemma 2.4 of Embrechts [11], which follows from Compton's result, also has conditions that may be easier to verify.

Forests of various sorts provide easy examples for the application of Theorem 2. These and other graphical examples are discussed by Compton [10] and by Knopfmacher and Knopfmacher [18] in their interesting papers. Inevitably, our examples overlap with these papers.

Example 1: For forests of trees, $C(x)$ enumerates trees and $A(x)$ enumerates forests. The verification of (a) is trivial. Crude asymptotic results on the number of trees are sufficient to prove convergence, (b), and (d); however, more refined asymptotics are usually available. Thus, when $C(R)$ converges, (a), (b), and (d) are easily verified and so a nonvanishing fraction of the forests consist of a single tree. Here are some observations about certain types of trees. 
- Unlabeled Trees: Let $T_{n}\left(\right.$ resp. $\left.t_{n}\right)$ be the number of unlabeled, $n$-vertex, rooted (resp. unrooted) trees of some type. See Harary, Robinson, and Schwenk [15] for information on estimating $T_{n}$ and $t_{n}$. In many cases, it can be shown that $T_{n} \sim$ $A n^{-3 / 2} R^{-n}$ and $t_{n} \sim b n^{-5 / 2} R^{-n}$ and so our theorem applies.

- Labeled Trees: In a variety of cases the exponential generating function for the rooted enumerator satisfies $T(x)=x \varphi(T(x))$. Under reasonable conditions on $\varphi$, one obtains $T_{n} \sim A n^{-3 / 2} R^{-n} n$ ! and so the theorem applies. Meir and Moon [19] have strengthened (f) by showing that, when $0 \leq \alpha<1$ and $d-\alpha n \sim \lambda n^{1 / 2}$, we have

$$
\operatorname{Pr}\left(X_{n}=d\right) \sim B_{1} e^{\lambda^{2} / 2 B_{2}} B_{3}^{d} B_{4}^{n},
$$

where the $B_{i}$ depend on $\varphi$ and $\alpha$. Compton [10,p. 76] points out that the generating functions need not be well behaved and gives the example of rooted trees where the root must not be the centroid of a tree with $2^{k}+1$ vertices. In this case, the circle of convergence is a natural boundary for $T(x)$, but Theorem 2 and Compton's Theorem 10 still apply.

- Plane Trees: Again, the theorem applies in many cases, but there are interesting cases that fall under UD. Then one can show that $c_{n} / c_{n}^{(2)} \rightarrow 0$ and so, by the result of Cameron [9] noted before Theorem 2, almost all forests of such trees contain more than one tree. We mention two examples. Various people have studied achiral trees; that is, rooted plane trees that are the same as their mirror images. In this case, $t_{n} \sim 2^{n} / \sqrt{\pi n}$. Odlyzko [20] studied the asymptotics of 2,3-trees, that is, trees in which each nonleaf node has 2 or 3 successors and all leaves are at the same depth. (The depth condition holds for each tree in the forest - not for the forest as a whole.)

In this case, the asymptotics is more complicated:

$$
t_{n} \sim R^{-n} u(\ln n) / n, \text { where } R<1 \text { and } u \text { is periodic. }
$$

Example 2: A map is an unlabeled graph embedded in a compact, boundaryless surface so that all faces are homeomorphic to discs. (The disc requirement implies that the graph is connected.) Various types of maps have been studied; e.g., all maps, 2-connected maps, Eulerian maps, and triangulations. A rooting procedure destroys symmetries. In many cases, it is known that the number of $n$-edged such maps is asymptotic to

$$
A B^{n} / n^{1+5 \chi / 4} \text { (unrooted case) or } 4 A B^{n} / n^{5 \chi / 4} \text { (rooted case) }
$$

where $\chi$ is the Euler characteristic of the surface, $B$ depends on the type of map, and $A$ depends on both the type of map and the surface. See [22] for a proof of (7) for the unrooted case and for further references.

Zvonkin [28, p. 290] remarks that it is sometimes necessary in physics to consider maps which are not connected. In that case, each component is embedded in a separate surface. Since generating functions are often not available, analytic methods cannot be applied; however, (7) allows us to apply Theorem 2 when the surfaces are all spheres. We omit details. The result can be extended to surfaces whose genuses have some fixed arbitrary 
sum: There are only finitely many combinations of nonspherical surfaces whose genuses add up to some fixed value. These can then be combined with an arbitrary number of spheres. For the nonspherical surfaces, we must consider sums of products of series whose asymptotics have the form (7). Consider a single term. If it is a product of $k$ series where the $i$ th has Euler characteristic $\chi_{i}$, then $2+\sum\left(\chi_{i}-2\right)$ is the same for all terms. Since $2-\chi_{i}>0, \sum n_{i}=n$, and

$$
\prod n_{i}^{-5 \chi_{i} / 4}=\left(\prod n_{i}^{5\left(2-\chi_{i}\right) / 4}\right)\left(\prod \frac{1}{n_{i}}\right)^{10 / 4}
$$

it is straightforward to show that the coefficients will grow fastest for the product containing only one factor. This result can then be convolved with the result for the all-spheres case. Again, we omit details.

We conclude with some observations that may be of interest but are not worth being called separate theorems. The bound in (b) is the value of $\rho$ in Theorem 2 .

Theorem 3. Suppose that $C_{n}>0$ for all sufficiently large $n$.

(a) If $\lim \sup _{n} c_{n}^{(2)} / c_{n}<\infty$, then $C(R)$ converges.

(b) If $\lim c_{n-1} / c_{n}=R$, then $\rho_{\text {sup }} \leq 1 / A(R)$. In particular, if $\lim c_{n-1} / c_{n}=R$ and $A(R)=\infty$, then $\rho=0$.

(c) In the labeled cases, $\sup _{n} c_{n}^{(2)} / c_{n} \leq M$ implies that $\rho_{\text {inf }} \geq M /\left(e^{M}-1\right)$.

(d) Monotonicity is not very informative: $C_{n} \geq 1$ for all $n$ makes $A_{n}$ monotonic and, even if $C_{n}$ is monotonic, $\rho$ may not exist, as (6) shows.

\section{Proof of Theorem 1 Part I: Only Listed Values Can Occur}

In this section we prove that only the values listed in Theorem 1 for $\left(\rho_{\text {inf }}, \rho_{\text {sup }}\right)$ can occur. The proof that these values actually do occur is deferred to the last section because it involves a series of fairly lengthy constructions and is not needed for the proof of the other theorems.

The case $R=0$ of Theorem 1 was done by Bell [3], who also showed that $\rho=1$ implies $R=0$, from which it follows that $\rho_{\text {inf }}<1$ when $R \neq 0$. To show that only the claimed values can occur, it suffices to prove the following lemma.

Lemma 1. When $C(R)$ diverges, $\rho_{\mathrm{inf}}=0$. When $R>0$ and $C(R)$ converges, $\rho_{\mathrm{sup}}>0$.

We require Theorem 3 of Stam [24]:

Theorem 4 (Stam). Let $g(x)$ be a power series with nonnegative coefficients, $g(0)=0$, and radius of convergence $R>0$. Let $\sum q_{n}(y) x^{n} / n !=\exp \{y g(x)\}$. Then

(i) If $g(R)$ converges, then $\lim \sup q_{n}(y) / q_{n}(1)>0$ for all $y>0$.

(ii) If $R=\infty$ or $g(R)$ diverges, then $\liminf q_{n}(y) / q_{n}(1)=0$ for $0 \leq y<1$. 
Proof (when $C(R)$ diverges): We prove $\rho_{\text {inf }}=0$ even when the $C_{n}$ are only required to be nonnegative real numbers. With the same values of $c_{n}$, the unlabeled $a_{n}$ is at least as large as the labeled value because the exponential in (2) contains more terms than in (1). Hence $\rho_{\text {inf }}=0$ for the unlabeled case will follow from the labeled. Apply Theorem 4(ii) with $g(x)=C(x)$. We have $q_{n}(1)=A_{n}$ and

$$
q_{n}(y)=n !\left[x^{n}\right](\exp (y C(x)))=n !\left[x^{n}\right](y C(x)+\ldots)=y C_{n}+\ldots
$$

Thus $q_{n}(y) / q_{n}(1) \geq y C_{n} / A_{n}=y \rho_{n}$. By Theorem 4(ii), the liminf of the left side is zero and so $\rho_{\text {inf }}=0$.

Proof (when $C(R)$ converges): It suffices to prove that $\rho=0$ is impossible.

We begin with the labeled case. Apply Theorem $4(\mathrm{i})$ with $g(x)=2 C(x)$ and $y=1 / 2$ to conclude that $\lim \sup q_{n}(1 / 2) / q_{n}(1)>0$. We proceed by contradiction, assuming that $\rho=0$. From $x A^{\prime}(x)=x C^{\prime}(x) A(x)$, we have

$$
\begin{aligned}
q_{n}(1 / 2)=a_{n} & =\frac{1}{n} \sum_{k=0}^{n} k c_{k} a_{n-k}=\sum_{k=0}^{n} \frac{k c_{k}}{n a_{k}} a_{k} a_{n-k} \\
& <\sum_{k<n^{1 / 2}} n^{-1 / 2} a_{k} a_{n-k}+\sum_{k \geq n^{1 / 2}}\left(c_{k} / a_{k}\right) a_{k} a_{n-k} \\
& =o(1) \sum_{k=0}^{n} a_{k} a_{n-k}=o(1) q_{n}(1),
\end{aligned}
$$

contradicting $\lim \sup q_{n}(1 / 2) / q_{n}(1)>0$.

We now consider the unlabeled case. Since the coefficients of $C(x)$ are nonnegative integers, $0<R<1$. Replacing $c_{n}$ with $c_{n}+1$ for all $n$ multiplies $A(x)$ by the partition generating function and so increases $a_{n}$ by at least the partition function $p_{n}$ and so does not increase $\rho_{\text {inf }}$. Hence we can assume that $a_{n} \geq p_{n}$ for all $n$. It follows that there is a function $N(z)$ such that $a_{n}>z$ whenever $n>N(z)$.

Let

$$
H(x)=\sum_{k=1}^{\infty} \frac{C\left(x^{k}\right)}{k} \quad \text { so } \quad h_{n}=\sum_{d \mid n} \frac{c_{n / d}}{d} .
$$

One easily has that $H(x)$ converges on the circle of convergence since $C(x)$ does. By case $\mathrm{LC}, \rho_{\text {sup }}>0$. Hence there is an $\epsilon>0$ and an infinite set $\mathcal{N}$ of positive integers such that $h_{n} / a_{n}>\epsilon$ for $n \in \mathcal{N}$. Suppose that $n \in \mathcal{N}$ and $n>2 N(z)$. Using $A^{\prime}(x)=H^{\prime}(x) A(x)$ we have

$$
a_{n}=\frac{1}{n} \sum_{k=1}^{n} k h_{k} a_{n-k} \geq h_{n}+\sum_{\substack{d \mid n \\ d>1}} \frac{h_{n / d} a_{n-n / d}}{d} \geq h_{n}+z \sum_{\substack{d \mid n \\ d>1}} \frac{h_{n / d}}{d} .
$$

Since $n \in \mathcal{N}$, it follows that $a_{n}<h_{n} / \epsilon$ and so

$$
\sum_{\substack{d \mid n \\ d>1}} \frac{h_{n / d}}{d}<h_{n}(1 / \epsilon-1) / z=o\left(h_{n}\right) \quad \text { as } n \rightarrow \infty \text { through } N .
$$


By Möbius inversion,

$$
c_{n}-h_{n}=\sum_{\substack{d \mid n \\ d>1}} \frac{\mu(d) h_{n / d}}{d} .
$$

Since $|\mu(d)| \leq 1, c_{n}-h_{n}=o\left(h_{n}\right)$ as $n \rightarrow \infty$ through $\mathcal{N}$. Hence $c_{n} / a_{n} \geq \epsilon$ for all sufficiently large $n \in \mathcal{N}$.

\section{Proof of Theorem 2}

We will show

$$
(\mathrm{c}) \Longleftrightarrow(\mathrm{d}) \Longrightarrow(\mathrm{f}) \Longrightarrow(\mathrm{e}) \Longrightarrow(\mathrm{c}) \text {. }
$$

Since (e) is contained in the last part of (f), the proof that (f) implies (e) is trivial.

Proof (of (c) and (d) equivalence): Note that for fixed $\omega$

$$
\sum_{k \leq \omega} c_{k} c_{n-k} \sim \sum_{k \leq \omega} c_{k} R^{k} c_{n}=(C(R)-E(\omega)) c_{n},
$$

where $E(\omega) \rightarrow 0$ as $\omega \rightarrow \infty$. By a diagonal argument, it follows that for all sufficiently slowly growing $\omega=\omega(n)$ we have

$$
\sum_{k \leq \omega} c_{k} c_{n-k} \sim C(R) c_{n}
$$

Since, for fixed $n$, the sum in (d) is monotonic decreasing in $\omega$, the equivalence of (c) and (d) follows.

Proof (that (d) implies (f)): We begin by proving the implication for case LC in three steps:

(i) $c_{n}^{(d)}<K^{d-1} c_{n}$ for some $K$ and all sufficiently large $n$.

(ii) $c_{n}^{(d)} \sim d C(R)^{d-1} c_{n}$ uniformly for $d \leq D(n)$, where $D(n) \rightarrow \infty$ suffciently slowly.

(iii) $a_{n} \sim A(R, 1) c_{n}$.

Equation (4) will then follow:

- $\left[y^{d}\right] A(x, y)=C(x)^{d} / d$ ! counts the number of structures having exactly $d$ components,

- (ii) tells us that $\left[x^{n}\right]\left(\frac{C(x)^{d}}{d !}\right) \sim \frac{C(R)^{d-1} c_{n}}{(d-1) !}$, which equals $\left[y^{d-1}\right] A(r, y) c_{n}$, and

- (iii) tells us that $c_{n} / a_{n} \sim A(R, 1)$.

We also obtain (5):

$$
a_{n} \mathrm{E}\left(X_{n}\right)=\left.\left[x^{n}\right] \frac{\partial A(x, y)}{\partial y}\right|_{y=1}=\left[x^{n}\right] C(x) e^{C(x)}=\sum\left[x^{n}\right]\left(\frac{C(x)^{d+1}}{d !}\right)
$$


THE EleCtronic Journal of COMBinatorics 7 (2000), \#R33

$$
\begin{aligned}
& \sim \sum_{0 \leq d<D(n)} \frac{(d+1) C(R)^{d} c_{n}}{d !}+O\left(\sum_{d \geq D(n)} \frac{K^{d} c_{n}}{d !}\right) \\
& \sim c_{n} \sum_{d \geq 0} \frac{(d+1) C(R)^{d}}{d !}=c_{n} e^{C(R)}(C(R)+1) \\
& \sim a_{n}(C(R)+1)
\end{aligned}
$$

and $\partial A(R, y) / \partial y=C(R) A(R, y)$.

We now prove (i). Let $b_{n}=c_{n}$ if $c_{n}>0$ and $b_{n}=1$ if $c_{n}=0$. Since $c_{n}>0$ when $n>N$, it follows that (b) and (d) hold for the $b_{n}$ 's. Hence (c) holds and so, since $b_{n}>0$ for all $n$, we have

$$
b_{n}^{(2)}<K b_{n} \text { for some } K \text { and all } n \geq 0 .
$$

Inducting on $d$, we have $b_{n}^{(d+1)}<K^{d} b_{n}$ because

$$
b_{n}^{(d+1)}=\sum_{k=0}^{n} b_{k} b_{n-k}^{(d)}<\sum_{k=0}^{n} b_{k} K^{d-1} b_{n-k}=K^{d-1} \sum_{k=0}^{n} b_{k} b_{n-k}<K^{d} b_{n}
$$

Since $b_{k} \geq c_{k}$ and $c_{n}=b_{n}$ for $n>N$, (i) is proved.

By a diagonal argument, is suffices to prove (ii) for fixed $d$, which we now do by induction on $d$. The case $d=1$ is trivial. By definition

$$
c_{n}^{(d+1)}=\sum_{k=0}^{n} c_{k}^{(d)} c_{n-k}
$$

We split the sum into three pieces for $n \rightarrow \infty$ and fixed large $\omega$ :

$$
\begin{aligned}
\sum_{k<\omega} c_{k}^{(d)} c_{n-k} & \sim \sum_{k<\omega} c_{k}^{(d)} R^{k} c_{n} & \text { by (b) } \\
\sum_{k<\omega} c_{n-k}^{(d)} c_{k} & \sim \sum_{k<\omega} d C(R)^{d-1} c_{n-k} c_{k} & \text { by induction } \\
& \sim d C(R)^{d-1} \sum_{k<\omega} c_{n} R^{k} c_{k} & \text { by (b) } \\
\sum_{k=\omega}^{n-\omega} c_{k}^{(d)} c_{n-k} & \leq K^{d-1} \sum_{k=\omega}^{n-\omega} c_{k} c_{n-k} & \text { by (i) }
\end{aligned}
$$

By a diagonal argument, if $\omega=\omega(n) \rightarrow \infty$ sufficiently slowly, the three sums are asymptotically $C(R)^{d} c_{n}, d C(R)^{d-1} C(R) c_{n}$ and $o\left(c_{n}\right)$, respectively. (The first two since $C(R)$ converges and the last by (d).) This completes the induction.

Step (iii) is simple: Since $A(x)=\sum C(x)^{d} / d$ !, we can apply (ii) to those $d<D(n)$ and (i) to those $d \geq D(n)$ to obtain $a_{n} \sim A(R) c_{n}=A(R, 1) c_{n}$.

In proving the implication for case UC, we shall need Schur's Theorem: 
Theorem 5 (Schur). If $F(x)$ and $G(x)$ are power series such that $f_{n-1} / f_{n} \rightarrow R, G(x)$ has larger radius of convergence than $R$, and $G(R) \neq 0$, then

$$
\left[x^{n}\right](F(x) G(x)) \sim G(R) f_{n} .
$$

A proof is given in Theorem 2 of [4].

Let

$$
L(x, y)=\sum C(x)^{k} y^{k} / k !=e^{y C(x)}
$$

and

$$
U(x, y)=\sum U_{k}(x) y^{k}=A(x, y) / L(x, y)
$$

Note that $U_{k}(x)$ is finite sum of weighted finite products and the factors in the products are of the form $C\left(x^{d}\right) / d$ with $d \geq 2$. It follows that $U_{k}(x)$ has radius of convergence at least $R^{1 / 2}$. This exceeds $R$ since $0<R<1$. The generating function for structures with exactly $d$ components is

$$
\left[y^{d}\right](L(x, y) U(x, y))=\sum_{k=1}^{d}\left(C(x)^{k} / k !\right) U_{d-k}(x)+U_{d}(x) .
$$

From (f) for case LC,

$$
\left[x^{n}\right]\left(C(x)^{k} / k !\right) \sim\left(C(R)^{k-1} /(k-1) !\right) c_{n} .
$$

By (b), the ratio of consecutive coefficients of $C(x)^{k} / k$ ! approaches $R$ and so, by Theorem 5 ,

$$
\left[x^{n}\right]\left(\left(C(x)^{k} / k !\right) U_{d-k}(x)\right) \sim\left(U_{d-k}(R) C(R)^{k-1} /(k-1) !\right) c_{n}
$$

Choose $S$ such that $R<S<R^{1 / 2}$. Since $U_{d}(x)$ has radius of convergence at least $R^{1 / 2}$, its coefficients are $o\left(S^{-n}\right)$ whereas, by (b), $c_{n}$ grows faster than $S^{-n}$. It follows that

$$
\begin{aligned}
{\left[x^{n}\right]\left(\sum_{k=1}^{d}\left(C(x)^{k} / k !\right) U_{d-k}(x)+U_{d}(x)\right) } & \sim c_{n} \sum_{k=1}^{d} U_{d-k}(R) C(R)^{k-1} /(k-1) ! \\
& =c_{n}\left[y^{d-1}\right]\left(U(R, y) e^{C(R) y}\right)=c_{n}\left[y^{d-1}\right] A(R, y) .
\end{aligned}
$$

To complete the proof of (4), we estimate $a_{n}$. We have $A(x)=A(x, 1)=L(x, 1) U(x, 1)$. From LC, the coefficients of $L(x, 1)$ are asymptotic to $e^{C(R)} c_{n}$. The radius of convergence of $U(x, 1)$ exceeds $R$ since, for $x<R^{1 / 2}$,

$$
\sum_{k \geq 2} \frac{C\left(x^{k}\right)}{k}=\sum_{k \geq 2}\left(\frac{C\left(x^{k}\right)}{x^{k}}\right)\left(\frac{x^{k}}{k}\right)<\sum_{k \geq 2}\left(\frac{C(R)}{R}\right)\left(\frac{R^{k / 2}}{k}\right)<\infty .
$$


Apply Theorem 5 to conclude that $a_{n} \sim c_{n} A(R)=c_{n} A(R, 1)$. The claim concerning $\rho$ is immediate:

$$
\rho_{n}=\operatorname{Pr}\left(X_{n}=1\right) \sim 1 / A(R, 1)=1 / A(R) .
$$

We now prove (5). Note that

$$
\begin{aligned}
a_{n} \mathrm{E}\left(X_{n}\right) & =\left.\left[x^{n}\right] \frac{\partial A(x, y)}{\partial y}\right|_{y=1} \\
& =\left[x^{n}\right] C(x) e^{C(x)} U(x, 1)+\left[x^{n}\right] \sum_{d>1} C\left(x^{d}\right) A(x, 1) .
\end{aligned}
$$

By (8), $\left[x^{n}\right] C(x) e^{C(x)} \sim c_{n} e^{C(R)}(C(R)+1)$. Since $U(x, 1)$ and $\sum_{d>1} C\left(x^{d}\right)$ converge for $x<R^{1 / 2}$, it follows from Theorem 5 that

$$
a_{n} \mathrm{E}\left(X_{n}\right) \sim c_{n} e^{C(R)}(C(R)+1) U(R, 1)+\sum_{d>1} C\left(R^{d}\right) a_{n} .
$$

Since $c_{n} e^{C(R)} U(R, 1)=c_{n} A(R, 1) \sim c_{n}$, the proof is complete.

Proof (that (e) implies (c)): We first prove (c) for case LC. Throughout, $\omega$ denotes of function of $n$ that goes to infinity in a sufficiently slow manner. From $A^{\prime}(x)=C^{\prime}(x) A(x)$ and the fact that $\rho \neq 0$ we have

$$
n a_{n}=\sum_{k=0}^{n} k c_{k} a_{n-k} \sim \sum_{k>\omega} k c_{k} a_{n-k} \sim \sum_{k>\omega} k \rho a_{k} a_{n-k},
$$

where we could neglect the terms less that $\omega$ as long as $\omega=o(n)$ because for $k \leq \omega$ and $n$ large

$$
k c_{k} a_{n-k} \leq \omega a_{k} a_{n-k}=o\left((n-k) a_{n-k} a_{k}\right)
$$

and $(n-k) a_{n-k} a_{k}$ is included in the second sum over $k>\omega$. Similarly, we can restore such terms to obtain

$$
\begin{aligned}
n a_{n} & \sim \rho \sum_{k=0}^{n} k a_{k} a_{n-k} \\
& =\frac{\rho}{2}\left(\sum_{k=0}^{n} k a_{k} a_{n-k}+\sum_{k=0}^{n}(n-k) a_{n-k} a_{k}\right) \\
& =\frac{n \rho}{2} \sum_{k=0}^{n} a_{k} a_{n-k} .
\end{aligned}
$$

Hence

$$
a_{n} \sim \frac{\rho}{2} \sum_{k=0}^{n} a_{k} a_{n-k}=\frac{\rho a_{n}^{(2)}}{2} .
$$


From (10) and a "diagonal" argument,

$$
\begin{aligned}
c_{n} & \sim \rho a_{n} \sim \frac{\rho^{2} a_{n}^{(2)}}{2} \\
& =\rho c_{n} \sum_{k<\omega} a_{k} \frac{c_{n-k}}{c_{n}} \frac{\rho a_{n-k}}{c_{n-k}}+\frac{1}{2} \sum_{k=\omega}^{n-\omega} c_{k} c_{n-k} \frac{\rho a_{k}}{c_{k}} \frac{\rho a_{n-k}}{c_{n-k}} \\
& \sim \rho c_{n} \sum_{k<\omega} a_{k} R^{k}+\frac{1}{2} \sum_{k=0}^{n} c_{k} c_{n-k}-c_{n} \sum_{k<\omega} c_{k} \frac{c_{n-k}}{c_{n}} \\
& \sim \rho c_{n} A(R)+L_{n} c_{n}-c_{n} C(R),
\end{aligned}
$$

where $L_{n}$ is defined by $\sum c_{k} c_{n-k}=2 L_{n} c_{n}$. Factoring out $c_{n}$, we see that

$$
1 \sim \rho A(R)+L_{n}-C(R) \text { and so } L_{n} \sim L=1+C(R)-\rho A(R) .
$$

Solving for $\rho A(R)$ gives

$$
\rho A(R)=1+C(R)-L .
$$

Now consider replacing $c_{n}$ by $2 c_{n}$ and $a_{n}$ by $a_{n}^{(2)}$. From (10), the hypotheses of the theorem are still valid provided we replace $\rho$ by $\rho^{2}$. Hence (11) still holds with the appropriate values for $A(R), C(R)$, and $L$, namely $A(R)^{2}, 2 C(R)$ and $2 L$. Thus

$$
\rho^{2} A(R)^{2}=1+2 C(R)-2 L .
$$

Equating this to the square of (11) we obtain $(1+\delta)^{2}=1+2 \delta$ where $\delta=C(R)-L$. Hence $\delta=0$.

We now prove the implication for the case UC. Since $\rho$ exists and is nonzero, it follows from (a) that $a_{n-1} / a_{n} \sim R$. Let $U(x, y)$ be as in $(9)$ and recall that $U(x, 1)$ converges for $x<R^{1 / 2}$. Applying Theorem 5 to $e^{C(x)}=A(x) / U(x, 1)$, we conclude that $\left[x^{n}\right] e^{C(x)} \sim a_{n} / U(R, 1)$. Hence

$$
\lim _{n \rightarrow \infty}\left(\frac{c_{n}}{\left[x^{n}\right] e^{C(x)}}\right) \quad \text { exists. }
$$

Regarding $C(x)$ as a generating function for labeled structures, we have just shown that (e) holds and so, case LC implies (c).

\section{Proof of Theorem 3}

Proof (of (a)): If $R=0$, there is nothing to prove. Otherwise, the hypothesis asserts that $\left[x^{n}\right] C(x)^{2} \leq\left[x^{n}\right] M C(x)$ for some $M<\infty$ and $n$ sufficiently large. Thus, for some polynomial $p, C(r)^{2} \leq M C(r)+p(r)$ for $0<r<R$. Divide by $C(r)$. We claim $p(r) / C(r)$ 
is bounded as $r \rightarrow R$. This is obvious if $R<\infty$. When $R=\infty$, it follows from the assumption that $C_{n}>0$ for sufficiently large $n$.

Proof (of (b)): Since $A(0)=1$, there is nothing to prove when $R=0$.

We now show that it suffices to deal with the labeled cases. In case UD, $A(R)=\infty$. Hence the result for case LD implies the result for case UD. In case UC, let

$$
b_{n}=\min \left(\left[x^{n}\right] e^{C(x)}, e^{C(R)} c_{n}\right) .
$$

It follows from case LC that $\lim \sup \left(c_{n} /\left[x^{n}\right] e^{C(x)}\right) \leq 1 / e^{C(R)}$. Hence $b_{n} \sim e^{C(R)} c_{n}$. From Theorem 5,

$$
\left[x^{n}\right]\left(B(x) \exp \left\{\sum_{k \geq 2} C\left(x^{k}\right) / k\right\}\right) \sim \exp \left\{\sum_{k \geq 2} C\left(R^{k}\right) / k\right\} b_{n} \sim A(R) c_{n} .
$$

Since $C\left(x^{k}\right)$ has nonnegative coefficients and $0 \leq b_{n} \leq\left[x^{n}\right] e^{C(x)}$,

$$
\left[x^{n}\right]\left(B(x) \exp \left\{\sum_{k \geq 2} C\left(x^{k}\right) / k\right\}\right) \leq a_{n} .
$$

Combining the last two displayed equations gives $\lim \sup c_{n} / a_{n} \leq 1 / A(R)$.

We now prove the labeled cases. Fix $0<r<R$. Let $K=K(n)<n / 2$ be such that (i) $c_{n-k} / c_{n} \geq r^{k}$ for $0 \leq k \leq K$ and (ii) $K(n) \rightarrow \infty$. This is possible since $c_{n-1} / c_{n} \sim R$. For every nonnegative integer $d$

$$
\left[x^{n}\right] C(x)^{d+1} \geq(d+1) \sum_{i_{1}+\cdots+i_{d} \leq K} c_{i_{1}} \cdots c_{i_{d}} r^{i_{1}+\cdots+i_{d}} c_{n} \sim(d+1) C(r)^{d} c_{n} .
$$

Hence

$$
\liminf \left[x^{n}\right]\left(\sum_{d<D} \frac{C(x)^{d+1}}{c_{n}(d+1) !}\right) \geq \sum_{d<D} \frac{C(r)^{d}}{d !} .
$$

Since $D$ is arbitrary, it follows that $\liminf \left[x^{n}\right] e^{C(x)} / c_{n} \geq e^{C(r)}$. Now let $r \rightarrow R$.

Proof (of (c)): If $M=\infty$, there is nothing to prove since $\frac{M}{e^{M}-1}=0$. Suppose $M$ is finite and regard $C(x)$ as a formal power series. We have $C(x)^{2} \leq M C(x)$, with the inequality understood coefficientwise. By induction on $k$ and the fact that $c_{n} \geq 0$, it follows that $C(x)^{k} \leq M^{k-1} C(x)$ and so

$$
A(x)=\sum_{k=0}^{\infty} C(x)^{k} / k ! \leq 1+\frac{e^{M}-1}{M} C(x) .
$$

The result follows.

Proof (of (d)): Define new enumerators for connected structures by $C_{n}^{*}=C_{n}-1$ and let $A_{n}^{*}$ be the associated enumerators for all structures. In the labeled case, $A(x)=e^{x} A^{*}(x)$ and so

$$
A_{n}=\sum_{k=0}^{n}\left(\begin{array}{l}
n \\
k
\end{array}\right) A_{k}^{*}
$$


Since $\left(\begin{array}{l}n \\ k\end{array}\right)$ is an increasing function of $n$, we are done. In the unlabeled case,

$$
A_{n}=\sum_{k=0}^{n} p_{n-k} A_{k}^{*}
$$

where $p_{n-k}$, the number of partitions of $n-k$, is an increasing function of $n$.

Equation (6) can be used to construct a monotonic $C_{n}$ for which $\rho$ does not exist. It is easily shown that $c_{n}$ is eventually monotonic. By changing enough initial values of $c_{n}$, we can guarantee that $C_{n}$ is monotonic for all $n$.

\section{Proof of Theorem 1 Part II: All Listed Values Do Occur}

As already noted, Bell [3] proved the $R=0$ case, so we assume $R>0$. Our proof consists of a variety of constructions which are dealt with in a series of lemmas. All our constructions produce integers $C_{n} \geq 0$ which are nonzero for sufficiently large $n$. Here is how the lemmas deal with the various parts:

- Lemma 2 proves all cases when $\rho_{\text {inf }}=0$ and $\rho_{\text {sup }}=1$.

- Lemma 5 proves the convergent case for $0 \leq \rho_{\text {inf }} \leq \rho_{\text {sup }}<1$.

- Lemma 6 proves the convergent case for $0<\rho_{\text {inf }}<\rho_{\text {sup }}=1$.

- Lemma 7 proves the divergent case when $\rho_{\text {sup }}<1$.

As always, assume that $C(x)$ and $A(x)$ are related by (1) and (2). Define

$$
\tau(n)= \begin{cases}1, & \text { in the unlabeled case } \\ n !, & \text { in the labeled case }\end{cases}
$$

Lemma 2. Fix $R>0$ subject to the constraints discussed at the beginning of Section 2. In all four cases $L C, U C, L D$ and $U D$, there are positive integers $C_{n}$ such that $C(R)$ has radius of convergence $R, \rho_{\mathrm{inf}}=0$, and $\rho_{\mathrm{sup}}=1$.

Proof: Set

$$
E_{n}= \begin{cases}{\left[\tau(n) / n^{2} R^{n}\right]} & \text { for the convergent cases, } \\ {\left[n ! /(\ln n)^{1 / 4}\right]} & \text { for the } R=\infty \text { case } \\ {\left[\exp \left(n^{3 / 4}\right)\right]} & \text { for the unlabeled } R=1 \text { case, } \\ {\left[\tau(n) / R^{n}\right]} & \text { for the remaining divergent cases. }\end{cases}
$$

We will set $C_{n}=1$ for most values of $n$. For the exceptional values of $n$ (which will be specified later), we set $C_{n}=E_{n}$. Since $C_{n} \geq 1$, it follows that $A_{n} \rightarrow \infty$. Because there will be infinitely many nonexceptional indices, we have $\rho_{\text {inf }}=0$.

We prove that, if only finitely many values of $n$ are exceptional, then $A_{n} / E_{n} \rightarrow 0$. Let $K$ be an integer such that $C_{n} \leq K$ for all $n$. In the labeled case with $r=1 / \ln n$,

$$
A_{n} / n ! \leq\left[x^{0}\right]\left(x^{-n} e^{K\left(e^{x}-1\right)}\right)<r^{-n} e^{K e^{r}}=\left(e^{K} / \ln n\right)^{n},
$$


and so $A_{n} / E_{n} \rightarrow 0$ in this case. In the unlabeled case, $A_{n}$ is bounded by the $K$-fold convolution of the partition function $p_{n}=\exp \left(O\left(n^{1 / 2}\right)\right)$ with itself. This convolution is bounded by $(n+1)^{K-1} p_{n}^{K}=\exp \left(O\left(n^{1 / 2}\right)\right)$, and so $A_{n} / E_{n} \rightarrow 0$.

Denote the indices of the exceptional $C_{n}$ by $n_{1}, n_{2}, \ldots$. Set $n_{1}=3$. Suppose $n_{i}$ has been chosen for $i<k$. Let $\tilde{A}_{n}$ be the values of $A_{n}$ computed using those exceptional values and $C_{n}=1$ otherwise. By the preceding paragraph, there is an $n>n_{k-1}+1$ so that $\tilde{A}_{n} / E_{n}<1 / k$. Choose such an $n$ for $n_{k}$ and note that, for $n=n_{k}$, the new value of $A_{n}$ is $E_{n}-1$ larger than $\tilde{A}_{n}$ since the only change to $C(x)$ was to increase it by $\left(E_{n}-1\right) x^{n} / \tau(n)$. Thus

$$
\frac{C_{n}}{A_{n}}=\frac{E_{n}}{A_{n}}=\frac{E_{n}}{\left(E_{n}-1\right)+\tilde{A}_{n}}>\frac{1}{1+1 / k} .
$$

Since we make no more changes in $C_{j}$ for $j \leq n_{k}$, it follows that $C_{n} / A_{n}$ will not change and so $\rho_{\text {sup }}=1$.

Lemma 3. Suppose that $\alpha>0, \xi>1, p>1, R>0$, and that $\mathcal{A}$ is an infinite set of positive integers forming an arithmetic progression. Then there are nonnegative integers $C_{n}$ such that

(i) $C_{n}=0$ for $n \notin \mathcal{A}$,

(ii) $C_{n} \sim \alpha \tau(n) / n^{p} R^{n}$ for $n \in \mathcal{A}$, and

(iii) $A(R)=\xi$.

Proof: Find an $N \in \mathcal{A}$ such that setting

$$
C_{n}= \begin{cases}\alpha \tau(n) / n^{p} R^{n}, & \text { if } n \geq N \text { and } n \in \mathcal{A}, \\ 0, & \text { otherwise }\end{cases}
$$

leads to $A(R) \leq \xi$. Increase $C_{N}$ so that $A(R)=\xi$. Call this sequence $C_{n}^{[N]}$. Let $l<k$ be two consecutive elements of $\mathcal{A}$ and suppose the sequence $C_{n}^{[l]}$ has been defined. Define the sequence $C_{n}^{[k]}$ by

$$
C_{n}^{[k]}= \begin{cases}C_{n}^{[l]}, & \text { for } n \neq l, k, \\ \left\lfloor C_{l}^{[l]}\right\rfloor, & \text { for } n=l, \\ C_{k}^{[l]}+x_{l}, & \text { for } n=k,\end{cases}
$$

where $x_{k}$ is chosen so that $A(R)$ will be unchanged. Thus $0 \leq x_{k}$ and, in the labeled case

$$
x_{k}=\left\{C_{l}^{[l]}\right\} \frac{R^{l} / l !}{R^{k} / k !}=o\left(k ! / k^{p} R^{k}\right)=o\left(C_{k}^{[k]}\right),
$$

where $\{z\}$ is the fractional part of $z$. In the unlabeled case, changing $C_{i}$ by $\delta$ changes $\ln A(R)$ by

$$
\sum_{k>0} \delta R^{i k} / k=-\delta \ln \left(1-R^{i}\right) .
$$


Hence

$$
x_{k}=\left\{C_{l}^{[l]}\right\} \frac{\ln \left(1-R^{l}\right)}{\ln \left(1-R^{k}\right)}=O\left(R^{l-k}\right)=O(1) .
$$

Thus $x_{k}=o\left(C_{k}^{[k]}\right)$. Note that $C_{n}^{[m]}$ changes for at most two values of $m$ : once for $m=n$ and once for the preceding element in $\mathcal{A}$. Let $C_{n}$ be value of $C_{n}^{[m]}$ for $m>n$.

Lemma 4. Suppose $a, b, d, \alpha, R$ are all greater than zero, $p, q$ are greater than one, and $a^{2}+2 a>b^{2}$. Then there are nonnegative integers $C_{n}$ with

$$
\begin{gathered}
C_{n} \sim \begin{cases}\frac{\alpha \tau(n)}{n^{p} R^{n}}, & \text { if } n \text { is even, } \\
\frac{\alpha d \tau(n)}{n^{q} R^{n}}, & \text { if } n \text { is odd, }\end{cases} \\
\sum_{n \geq 0} \frac{A_{2 n} R^{2 n}}{\tau(2 n)}=1+a, \quad \text { and } \quad \sum_{n \geq 0} \frac{A_{2 n+1} R^{2 n+1}}{\tau(2 n+1)}=b,
\end{gathered}
$$

where $A(x)$ is given by (1) or (2).

Proof: The last displayed equations are equivalent to

$$
A(R)=1+a+b \quad \text { and } \quad A(-R)=1+a-b .
$$

Call these values $A^{+}$and $A^{-}$, respectively. Since

$$
A^{+} A^{-}=(1+a+b)(1+a-b)=(1+a)^{2}-b^{2}>1,
$$

it follows by Lemma 3 that we can find $\tilde{C}_{n}$ vanishing at odd $n$ and asymptotic to $\alpha \tau(n) / n^{p} R^{n}$ at even $n$ such that $\tilde{A}(R)=\sqrt{A^{+} A^{-}}$. Since $A^{+} / A^{-}>1$, it again follows that we can find $\hat{C}_{n}$ vanishing at even $n$ and asymptotic to $\alpha d \tau(n) / n^{q} R^{n}$ at odd $n$ such that $\hat{A}(R)=\sqrt{A^{+} / A^{-}}$. Let $C_{n}=\tilde{C}_{n}+\hat{C}_{n}$. Then $A(x)=\tilde{A}(x) \hat{A}(x)$ and so

$$
A(R)=\tilde{A}(R) \hat{A}(R)=A^{+} \quad \text { and } \quad A(-R)=\tilde{A}(-R) \hat{A}(-R)=\tilde{A}(R) / \hat{A}(R)=A^{-} .
$$

Lemma 5. Suppose $R>0,1>\rho_{\mathrm{sup}} \geq \rho_{\mathrm{inf}} \geq 0$ and $\rho_{\mathrm{sup}}>0$. In the unlabeled case, also suppose $R<1$. Then there are integers $C_{n}$ such that function $C(x)$ has radius of convergence $R, C(R)$ converges, $\lim \inf _{n \rightarrow \infty} C_{n} / A_{n}=\rho_{\text {inf }}$, and $\lim \sup _{n \rightarrow \infty} C_{n} / A_{n}=\rho_{\text {sup }}$.

Proof: We begin by choosing values to use in Lemma 4 , according as whether $\rho_{\text {inf }}=0$ or not.

Suppose $\rho_{\text {inf }}=0$. Let $d=1, a=\left(1 / \rho_{\text {sup }}\right)-1, \alpha=1, p=2, q=3$ and $b=a$.

Now suppose $\rho_{\text {inf }}>0$. Let $\alpha=1, p=q=2, \mu=\left(1 / \rho_{\text {sup }}\right)-1$ and $\nu=\left(1 / \rho_{\text {inf }}\right)-1$. Note that $\nu \geq \mu>0$. If $\mu=\nu$, let $d=1$ and $a=b=\mu / 2$; otherwise, we claim that for sufficiently small $d$ there exist $a, b>0$ such that $a(a+2)>b^{2}$ and

$$
\left(\begin{array}{cc}
1 & d \\
1 & 1 / d
\end{array}\right)\left(\begin{array}{l}
a \\
b
\end{array}\right)=\left(\begin{array}{l}
\mu \\
\nu
\end{array}\right)
$$


To see this, solve and note that, as $d \rightarrow 0$, we have $a \sim \mu$ and $b \sim(\nu-\mu) d$. Choose such a small $d$.

Using the values in the preceding two paragraphs, apply Lemma 4 . Choose $k$ such that $a_{k}>0$, and $a_{k-1}>0$ and suppose $n>2 k$. Let $H(x)=\ln A(x)$. From $A^{\prime}(x)=H^{\prime}(x) A(x)$, we have

$$
n a_{n}=\sum(n-k) h_{n-k} a_{k}
$$

Hence

$$
n a_{n}=\sum(n-i) h_{n-i} a_{i} \geq(n / 2)\left(a_{k} c_{n-k}+a_{k-1} c_{n-k+1}\right)=\Theta\left(1 / n R^{n}\right) .
$$

Replacing $C_{n}$ with larger values that are asymptotic to $\alpha \max (1, d) \tau(n) / n^{2} R^{n}$ increases the value of $a_{n}$ and allows us to use Theorem 2 to conclude that the new $a_{n}$, and hence the old $a_{n}$, are $O\left(1 / n^{2} R^{n}\right)$. Combining this with (14), we have

$$
A_{n}=\Theta\left(\tau(n) / n^{2} R^{n}\right) .
$$

In the labeled case, $h_{n}=c_{n}$ and in the unlabeled case

$$
h_{n}=\sum_{d \mid n} \frac{c_{d}}{n / d}=c_{n}+O\left(\sum_{d \leq n / 2} R^{-d}\right)=c_{n}+O\left(R^{-n / 2}\right),
$$

and so $h_{n} \sim c_{n}$. From this and (13), it follows easily that

$$
a_{n} \sim c_{n} \sum_{k \text { even }} a_{k} R^{k}+c_{n-1} \sum_{k \text { odd }} a_{k} R^{k-1}=c_{n}(1+a)+c_{n-1} \frac{b}{R} .
$$

We now use this, treating $\rho_{\text {inf }}>0$ and $\rho_{\text {inf }}=0$ separately. For $\rho_{\text {inf }}>0$

$$
\frac{a_{2 n}}{c_{2 n}} \sim(1+a)+\frac{c_{2 n-1} b}{R c_{2 n}} \sim 1+a+\frac{b}{d}=1+\mu=\frac{1}{\rho_{\text {inf }}}
$$

and, similarly, $a_{2 n+1} / c_{2 n+1} \sim 1 / \rho_{\text {sup }}$. For $\rho_{\text {inf }}=0$, we have $a_{2 n} / c_{2 n} \sim 1+a=1 / \rho_{\text {sup }}$ from (16), and, from (15) and the asymptotics for $C_{2 n+1}, C_{2 n+1} / A_{2 n+1} \rightarrow 0$.

Lemma 6. In both the labeled and unlabeled convergent cases, if $0<\lambda<1$, there are integers $C_{n}$ tending to infinity such that $C(x)$ has radius of convergence $R, \rho_{\mathrm{inf}}=\lambda$, and $\rho_{\text {sup }}=1$.

Proof: Let $\zeta_{n}=\tau(n) R^{-n} / n^{\nu(n)}$ where

$$
\nu(n)=3-\sum_{i=1}^{\log _{3}\left(\log _{3} n\right)} 2^{-i}
$$

Find an $N>0$ such that setting

$$
C_{n}= \begin{cases}\zeta_{n}, & \text { for } n \geq N \\ 0, & \text { otherwise }\end{cases}
$$


leads to $A(R) \leq 1 / \lambda$. Increase $C_{N}$ so that $A(R)=1 / \lambda$. Call this sequence $C_{n}^{[N]}$. Proceed as in the proof of Lemma 3 so as to obtain a sequence of integers $C_{n}$ with $C_{n} \sim \zeta_{n}$ and $A(R)=1 / \lambda$. Clearly $C(x)$ has radius of convergence $R$ and converges at $R$.

We now show that $\rho_{\text {inf }}=1 / A(R)$. Let $H(x)=\ln A(x)$. As in the proof of Lemma 5, $h_{n} \sim c_{n}$. Let $\hat{h}_{k}=h_{k} k^{\nu(k)-\nu(n)}$. From $n a_{n}=\sum k h_{k} a_{n-k}$, we have

$$
n a_{n} \leq \sum k \hat{h}_{k} a_{n-k} \sim n \hat{h}_{n} A(R) \sim n c_{n} A(R)
$$

Hence $\rho_{\text {inf }} \geq 1 / A(R)$. Letting $n \rightarrow \infty$ through $n=3^{3^{k}}-1$, one easily sees that $n a_{n} \sim$ $h_{n} A(R)$ and so $\rho_{\text {inf }}=1 / A(R)$.

We now show that $\rho_{\text {sup }}=1$. Let $n \rightarrow \infty$ through $n=3^{3^{k}}$. For such an $n$, let $\tilde{c}_{n}=c_{n} / n^{\nu(n-1)-\nu(n)}$ and let $\tilde{a}_{n}$ be the value of $a_{n}$ obtained when $c_{n}$ is replaced by $\tilde{c}_{n}$. By the argument in the preceding paragraph, $\tilde{c}_{n} / \tilde{a}_{n} \rightarrow \rho_{\text {inf. }}$. Since $A(x)=e^{H(x)}$ and since $H(x)$ and $\tilde{H}(x)+\left(c_{n}-\tilde{c}_{n}\right) x^{n}$ agree through terms of degree $n$, we have $a_{n}=\tilde{a}_{n}+\left(c_{n}-\tilde{c}_{n}\right)$. Thus

$$
\frac{c_{n}}{a_{n}}=\frac{1}{1+\left(\tilde{a}_{n} / \tilde{c}_{n}-1\right) \tilde{c}_{n} / c_{n}} \sim \frac{1}{1+\left(1-1 / \rho_{\text {inf }}\right) \tilde{c}_{n} / c_{n}} .
$$

Since $n=3^{3^{k}}$ and $\nu(n)-\nu(n-1)=2^{-k}$, we have

$$
\frac{\tilde{c}_{n}}{c_{n}} \sim\left(3^{3^{k}}\right)^{-2^{-k}}=3^{-(3 / 2)^{k}} \rightarrow 0
$$

Thus $\lim _{k \rightarrow \infty} c_{n} / a_{n}=1$ where $n=3^{3^{k}}$.

Lemma 7. Suppose $R>0$ and $0<\alpha<1$. In the the labeled and unlabeled cases, there are integers $C_{n}>0$ such that the function $C(x)$ has radius of convergence $R, C(R)$ diverges, and $\rho_{\text {sup }}=\alpha$.

Proof: Define $\beta=(1-1 / \alpha)^{-1}$ and

$$
R_{n}= \begin{cases}(\ln n)^{n / 2}, & \text { if } R=\infty \\ \exp \left(-n^{-1 / 3}\right), & \text { if unlabeled and } R=1 \\ R, & \text { otherwise. }\end{cases}
$$

Let $C_{n}^{[1]}=1$ for all $n>1$ and let $C_{1}^{[1]}$ be any integer greater than $1 / \alpha R$. If $C_{n}^{[k-1]}$ has been defined for all $n$, let $A_{n}^{[k-1]}$ be the corresponding $A(x)$ sequence and let

$$
C_{n}^{[k]}= \begin{cases}C_{n}^{[k-1]}, & \text { if } n \neq k \\ C_{k}^{[k-1]}, & \text { if } n=k \text { and } A_{k}^{[k-1]} \geq \tau(k) / R_{k}^{k} \\ \left\lceil\beta \tau(k) A_{k}^{[k-1]}\right\rceil, & \text { otherwise. }\end{cases}
$$

Since $C_{n}^{[k]}$ is unchanging for $k>n$, we define $C_{n}=C_{n}^{[n+1]}$. Let $\mathcal{K}$ be the set of $k$ for which the third alternative is used. We will show that 
(a) $\mathcal{K}$ is infinite.

(b) The radius of convergence of $C(x)$ is at least $R$.

(c) $\lim _{n \rightarrow \infty} C_{n} / A_{n}=\alpha$, where the limit is taken through $n \in \mathcal{K}$.

(d) $C(R)=\infty$.

The lemma follows immediately from these claims.

To prove (a), it suffices to show that $\lim _{n \rightarrow \infty} A_{n}^{[k]} R_{n}^{n} / \tau(n)=0$. In the labeled case, $A^{[k]}(x)=\exp \left(p(x)+e^{x}-1\right)$, where $p(x)$ is a polynomial with no constant term. Proceeding as in (12), we obtain

$$
A_{n}^{[k]} / n !<\exp (p(\ln n)) \times(e / \ln n)^{n}=o\left(1 /(\ln n)^{n / 2}\right) .
$$

By the definition of $R_{n}$, this completes the proof of (a) for the labeled case. In the unlabeled case, $A^{[k]}(x)$ equals the partition generating function $p(x)$ times finitely many factors of the form $\left(1-x^{i}\right)^{-b_{i}}$. Hence there are constants $B$ and $l$ depending on the $b_{i}$ such that

$$
\begin{aligned}
A_{n}^{[k]} & \leq\left[x^{n}\right]\left(\frac{p(x)}{(1-x)^{l}}\right) \leq\left[x^{n}\right]\left(\frac{1}{(1-x)^{l}} \frac{p_{n}}{1-x}\right) \\
& \leq n^{l+1} p_{n}=o\left(\exp \left(n^{2 / 3}\right)\right)=O\left(\left(\exp \left(-n^{-1 / 3}\right)\right)^{-n}\right) .
\end{aligned}
$$

This completes the proof of (a).

From the definition of $C_{n}^{[k]}$, it follows that $C_{n}^{[k]}<1+\beta \tau(n) / R_{n}^{n}$ for all $k$ and $n$. This proves (b).

To prove (c) we use $A_{k}^{[k]}=A_{k}^{[k-1]}-C_{k}^{[k-1]}+C_{k}^{[k]}$. When $k \in \mathcal{K}, C_{k}^{[k-1]}=1 / \tau(k)$ and $C_{k}^{[k]}$ differs from $\beta A_{k}^{[k]}$ by less than 1. Hence $C_{k}^{[k]} / A_{k}^{[k]} \sim \beta /(1+\beta)=\alpha$.

Finally, we prove that $C(R)=\infty$. It suffices to show that the middle condition in (17) holds infinitely often. Suppose not. It follows from (c) that $\rho$ exists and equals $\alpha$. With $H(x)=\ln A(x)$ we have $A^{\prime}(x)=H^{\prime}(x) A(x)$ and so

$$
n a_{n}=\sum_{k=1}^{n} k h_{k} a_{n-k} \geq(n-1) h_{n-1} a_{1} \geq(n-1) c_{n-1} a_{1}=(n-1) c_{n-1} C_{1}
$$

Hence $a_{n} / c_{n} \geq C_{1}(1-1 / n) c_{n-1} / c_{n}$ and so

$$
\frac{1}{\alpha}=\lim _{n \rightarrow \infty} \frac{a_{n}}{c_{n}} \geq C_{1} \limsup _{n \rightarrow \infty} \frac{c_{n-1}}{c_{n}}
$$

Since $\lim \sup _{n \rightarrow \infty}\left|b_{n-1} / b_{n}\right|$ is at least the radius of convergence of a power series $\sum b_{n} x^{n}$ and since $C_{1}>1 /(\alpha R)$, we have $1 / \alpha>(1 /(\alpha R)) R$, a contradiction. 


\section{References}

[1] R. Arratia, A. D. Barbour, and S. Tavaré, Notices AMS 44 (1997) 903-910.

[2] J. S. Beissinger, The enumeration of irreducible combinatorial objects, J. Combin. Theory, Ser. A 38 (1985) 143-169.

[3] J. P. Bell, When structures are almost surely connected, Electron. J. Combin. submitted.

[4] E. A. Bender, Asymptotic methods in enumeration, SIAM Rev. 16 (1974), 485-515.

[5] E. A. Bender, P. J. Cameron, A. M. Odlyzko, and L. B. Richmond, Connectedness, classes and cycle index, Combin., Probab. and Comput. 8 (1999) 31-43.

[6] S. Burris, Spectrally determined first-order limit laws, DIMACS Series in Discrete Math. and Theoret. Comput. Science 33 (1997)33-52.

[7] S. Burris, K. Compton, A. Odlyzko, and B. Richmond, Fine spectra and limit laws II. First-order 0-1 laws, Canad. J. Math. 49 (1997) 641-652.

[8] S. Burris and A. Sárközy, Fine spectra and limit laws I. First-order laws, Canad. J. Math. 49 (1997) 468-498.

[9] P. J. Cameron, On the probability of connectedness, Discrete Math. 167/168 (1997) $175-187$.

[10] K. J. Compton, Some methods for computing component distribution probabilities in relational structures, Discrete Math. 66 (1987) 59-77.

[11] P. Embrechts, The asymptotic behaviour of series and power series with positive coefficients, Med. Konink. Acad. Wetensch. (Brussels) 45 (1983) 41-61.

[12] P. Flajolet and M. Noy, Analytic combinatorics of non-crossing partitions, INRIA Rpt. No. 3196 (1997).

[13] P. Flajolet and M. Soria, Gaussian limiting distributions for the number of components in combinatorial structures, J. Combin. Theory, Ser. A 53 (1990) 165-182.

[14] X. Gourdon, Largest component in random combinatorial structures, Discrete Math. 180 (1998) 185-209.

[15] F. Harary, R. W. Robinson, and A. J. Schwenk, Twenty-step algorithm for determining the asymptotic number of trees of various species, J. Austral. Math. Soc., Ser. A 20 (1975) 483-503.

[16] W. K. Hayman, A generalisation of Stirling's formula, J. Reine Angew. Math. 196 (1956) 67-95.

[17] H.-K. Hwang, A Poisson * geometric convolution law for the number of components in unlabelled combinatorial structures, Combin., Probab. and Comput. 7 (1998) 89-110.

[18] A. Knopfmacher and J. Knopfmacher, Arithmetical semigroups related to trees and polyhedra, J. Combin. Theory, Ser. A 86 (1999) 85-102.

[19] A. Meir and J. W. Moon, The asymptotic behaviour of coefficients of certain generating functions, Europ. J. Combin. 11 (1990) 581-587. 
[20] A. M. Odlyzko, Periodic oscillations of coefficients of power series that satisfy functional equations, Adv. Math. 44 (1982) 180-205.

[21] H. J. Prömel, Counting unlabeled structures, J. Combin. Theory, Ser. A 44 (1987) 83-93.

[22] L.B. Richmond and N.C. Wormald, Almost all maps are asymmetric, J. Combin. Theory, Ser. B 63 (1995) 1-7.

[23] W. Rudin, Limits of ratios of tails of measures, Ann. Probab. 1 (1973) 982-994.

[24] A.J. Stam, Polynomials of binomial type and compound Poisson Processes, J. Math. Anal. Appl. 130 (1988) 493-508.

[25] E. M. Wright, A relationship between two sequences, Proc. London Math. Soc. (iii) 17 (1967) 296-304.

[26] E. M. Wright, A relationship between two sequences III, J. London Math. Soc. 43 (1968) 720-724.

[27] E. M. Wright, Asymptotic relations between enumerative functions in graph theory, Proc. London Math. Soc. (iii) 20 (1970) 558-572.

[28] A. Zvonkin, Matrix integrals and map enumeration: An accessible introduction, Mathl. Comput. Modeling 26 (1997) 281-304. 